\title{
RESULTADOS MATERNOS E NEONATAIS DA ASSISTÊNCIA EM CASA DE PARTO NO MUNICÍPIO DO RIO DE J ANEIRO
}

\author{
Maternal and neonatal assistance results at Birthing Center in the municipality of Rio \\ de J aneiro
}

\author{
Resultados maternos y neonatales de la asistencia en casa de nacimiento en el \\ municipio de Rio de J aneiro
}

Adriana Lenho de Figueiredo Pereira ${ }^{1}$

Monique da Silva Ferreira Gouveia ${ }^{4}$
Tamara Rubia Lino de Lima²

Mariana Santana Schroeter ${ }^{3}$

\section{RESUMO}

0 objetivo deste estudo foi descrever os resultados maternos e neonatais da assistência na Casa de Parto David Capistrano Filho. Pesquisa exploratório-descritiva, com abordagem quantitativa, que analisou 458 prontuários dos partos normais e nascimentos no período de janeiro de 2008 a dezembro de 2009. As parturientes eram mulheres jovens, de 15 a 25 anos de idade $(66,6 \%)$, e nulíparas (55\%). Durante o trabalho de parto, elas permaneceram com o acompanhante $(94,1 \%)$ e receberam cuidados para 0 relaxamento e o conforto. A taxa de episiotomia foi de 2,4\%. Não houve óbitos maternos e neonatais. Os casos de asfixia neonatal representaram $0,2 \%$ dos nascidos vivos. As transferências para o hospital corresponderam a 2,8\% das mulheres no pós-parto e $8,5 \%$ entre os neonatos. A maioria dos resultados encontrados foi semelhante aos descritos nas pesquisas brasileiras e internacionais acerca da assistência em centros de parto.

Palavras-chave: Assistência de enfermagem. Parto normal. Centros Independentes de Assistência à Gravidez e ao Parto. Enfermagem obstétrica.

\begin{abstract}
The purpose of this study was to describe the maternal and neonatal assistance results in the Casa de Parto David Capistrano Filho Birthing Center. An exploratory and descriptive research with a quantitative approach, which were analyzed 458 patients' files data of normal delivery and births in period from January 2008 to December 2009. The pregnants were young women, from 15 to 25 years old (66.6\%), and nulliparous (55\%). During labor, they remained with the companion (94.1\%) and received care to relaxation and comfort. The episiotomy rate was $2.4 \%$. There were no maternal and neonatal deaths. The cases of neonatal asphyxia accounted for $0.2 \%$ of live births. Transfers rate to hospital corresponded to $2.8 \%$ of women postpartum and $8.5 \%$ among newborns. Most results were similar to those described in Brazilian and internationals researches about birth centers assistance.
\end{abstract}

Keywords: Nursing care. Natural childbirth. Birthing centers. Obstetrical nursing.

\section{Resumen}

El objetivo de este estudio fue describir los resultados maternos y neonatales de la asistencia en la Casa de Parto David Capistrano Filho. Investigación exploratoria y descriptiva con enfoque cuantitativo, que analizó 458 registros clínicos de los partos normales y nacimientos en el período de Enero de 2008 a Diciembre de 2009. Las mujeres embarazadas eran mujeres jóvenes, entre $15 y$ 25 años de edad (66,6\%), y nulíparas (55\%). Durante el trabajo de parto, ellas recibieron dieta oral (100\%), permanecieron con el acompañante $(94,1 \%)$ y recibieron cuidados para la relajación y confort. La tasa de episiotomía fue del 2,4\%. No hubo óbitos maternos y neonatales. Los casos de asfixia neonatal representaron $0,2 \%$ de los neonatos. Los traslados para el hospital correspondieron a 2,8\% de las mujeres en el postparto y a $8,5 \%$ entre los recién nacidos. La mayoría de los resultados fueron similares a los descritos en las investigaciones brasileñas e internacionales a cerca de la asistencia en centros de nacimientos.

Palabras clave: Atención de enfermería. Parto normal. Centros Independientes de Asistencia al Embarazo y al Parto. Enfermería obstétrica

\footnotetext{
${ }^{1}$ Enfermeira obstétrica. Doutora em Enfermagem. Professora Adjunta do Departamento de Enfermagem Materno-Infantil, Faculdade de Enfermagem, Universidade do Estado do Rio de Janeiro-RJ. Brasil. E-mail: adrianalenho.uerj@gmail.com; ${ }^{2}$ Acadêmica de Enfermagem, Bolsista de Iniciação CientíficaCNPq, Faculdade de Enfermagem, Universidade do Estado do Rio de Janeiro- RJ. Brasil. Email: tamararubia.uerj@gmail.com; ${ }^{3}$ Acadêmica de Enfermagem, Bolsista de Iniciação Científica-UERJ, Faculdade de Enfermagem, Universidade do Estado do Rio de Janeiro- RJ. Brasil. E-mail: mariana.uerj.2010.1@gmail.com; ${ }^{4}$ Acadêmica de Enfermagem, voluntária da Pesquisa de Iniciação Científica, Faculdade de Enfermagem, Universidade do Estado do Rio de Janeiro-RJ. Brasil. E-mail: monique.gouveia-enf@hotmail.com; ${ }^{5}$ Acadêmica de Enfermagem, voluntária da Pesquisa de Iniciação Científica, Faculdade de Enfermagem, Universidade do Estado do Rio de Janeiro-RJ. Brasil. E-mail: sasadamazio@hotmail.com
} 


\section{INTRODUÇÃO}

Nos Estados Unidos, as experiências de instituições diferenciadas do hospital para assistência ao parto, como casa ou centro de parto, surgiram nos anos 1940. 0 primeiro centro foi o "La Casita" do Medical Mission Sisters em Santa Fé, Estado do Novo México, que era dirigido por enfermeiras obstétricas. Outra experiência foi em Raymondsville, no Texas, nos anos de 1970, como centro de parto vinculado à atenção primária' .

Posteriormente, em 1975, surgiu o primeiro centro de parto independente ou isolado do hospital na cidade de Nova York, que foi fundado por uma equipe de enfermeiras obstétricas e médicos. 0 sucesso dessa iniciativa, com altos índices de satisfação materna, bons resultados perinatais e 0 apoio federal para a formação de enfermeiras obstétricas americanas, fez com que outros centros de parto isolados fossem abertos no país'.

No entanto, surgiram questionamentos acerca da segurança assistencial nesses centros de parto, que motivaram a Associação Americana de Centros de Parto (AABC) e o Colégio Americano de Enfermeiras Obstétricas (ACNM), entre outras organizações do país, a compilar um grande corpo de pesquisas, que evidenciou bons resultados acerca da segurança assistencial em um centro de parto credenciado. Em 2009, havia 195 centros de partos em funcionamento no país'.

No Reino Unido também houve movimento de retorno aos centros de parto a partir dos anos de 2000. De 1970 até o ano 2000 houve predominância dos nascimentos hospitalares, que provocou considerável diminuição destes centros ou unidades dirigidas por obstetrizes ou enfermeiras obstétricas, de $13 \%$ em 1970 para $3 \%$ em $2000^{2}$.

0 estímulo desses cenários assistenciais surge como uma alternativa para cessar a tendência de elevação das intervenções e cesarianas nos nascimentos hospitalares. Na Irlanda, ocorreu aumento de $81,5 \%$ no percentual de cesarianas no período de 1991 a 2000, de 11,8\% para 21,3\% dos partos. Também na Inglaterra houve considerável elevação dessa cirurgia, passando de $9 \%$ em 1980 para $21 \%$ dos nascimentos no ano de $2001^{2}$.

Na Holanda, há uma tradição de parto domiciliar e a taxa de cesarianas foi de $13,7 \%$ no ano de 2003. As parteiras licenciadas atendem cerca de $70 \%$ dos nascimentos no país. Dentre os nascimentos atendidos por estas profissionais, quase $60 \%$ foram resultantes de partos domiciliares e os demais ocorreram em instituiç̃es, nos hospitais e em pequenas unidades de saúde comunitárias, que são os centros de parto. Estes centros foram criados em 1979 e são instituições de curta permanência e têm ambiente semelhante ao doméstico. Os casos com complicações maternas ou perinatais são referenciados para o nível secundário, que dispõe de médicos especialistas ${ }^{3}$.

Na Austrália, os centros de parto foram instituídos durante os anos de 1990. Em 1997 estavam em funcionamento
24 centros. Esta expansão tem sido atribuída à revisão ministerial dos serviços maternos, que preconizou modelos alternativos ao cuidado medicalizado presente na cultura obstétrica desse país4.

No Brasil, o Ministério da Saúde instituiu os Centros de Parto Normal (CPN) para o atendimento de mulheres com gestações de baixo risco em 1999. Os CPN podem ser unidade assistencial situada dentro, anexa ou isolada do hospital, onde atuam as enfermeiras obstétricas ${ }^{5}$. Há uma tendência de denominar Casa de Parto os CPN que são fisicamente distanciados do hospital. Geralmente, a Casa de Parto está localizada em uma comunidade que pertence a um distrito de saúde e possui um hospital como referência para os casos de risco obstétrico e perinatal.

Essa iniciativa ministerial viabilizou a implantação dos CPN nos sistemas de saúde municipais. No entanto, o quantitativo destes Centros no país é muito reduzido. Atualmente, existem apenas três CPN distanciados do hospital e vinculados à rede pública de saúde, no Distrito Federal e nas cidades de São Paulo e do Rio de Janeiro.

A Secretaria Municipal de Saúde e Defesa Civil do Rio de Janeiro (SMSDC/RJ) instituiu uma Casa de Parto na rede pública de saúde em março de 2004, que foi denominada Casa de Parto David Capistrano Filho (CPDCF).

A CPDCF atende gestantes no pré-natal, no parto e no pós-parto, bem como oferece pronto-atendimento daquelas que apresentam intercorrência obstétrica. 0 atendimento ao parto normal é vinculado ao acompanhamento pré-natal realizado na instituição, desde a primeira consulta ou quando estas mulheres são referenciadas da atenção básica e estão com gravidez na $34^{\mathrm{a}}$ semana ou menos. A assistência à mulher $\mathrm{e}$ aos recém-nascidos está sob a responsabilidade das enfermeiras obstétricas, e os casos que requerem acompanhamento médico são encaminhados para uma maternidade pública localizada nas adjacências.

Devido à implantação desse novo cenário de atuação da enfermeira obstétrica na rede pública de saúde do município do Rio de Janeiro e a necessidade de se investigar sobre a assistência materna e neonatal prestada nesse cenário, foi proposta a presente pesquisa que objetivou descrever os resultados maternos e neonatais da assistência na Casa de Parto David Capistrano Filho.

\section{MÉTODO}

Estudo de abordagem quantitativa, exploratória e descritiva, tendo como fonte de dados as informações registradas nos prontuários atinentes aos partos normais atendidos na CPDCF, no período de janeiro de 2008 a dezembro de 2009.0 estudo obteve parecer favorável do Comitê de Ética em Pesquisa da SMSDC/RJ, protocolo $n^{0}$ 202A/2010.

A CPDCF está situada no bairro de Realengo, distante cerca de $40 \mathrm{~km}$ do centro da cidade, onde predomina uma 
população mais empobrecida e com menor acesso aos serviços de saúde em comparação àquela que residente em bairros da zona central e sul da cidade. Ela é unidade pública de saúde aberta 24 horas.

A CPDCF tem estrutura física plana assemelhada a uma casa, dispondo de ambientes coloridos com decoração harmoniosa e suave. Possui consultório de atendimento prénatal, sala de preparo de medicações e três suítes. Estas suítes têm uma ampla cama de casal, berço, recursos para promoção do confor to e relaxamento durante o trabalho de parto, como a bola de Bobath, banquinho e banheira, entre outros, bem como instrumentais indispensáveis para a assistência ao parto.

Dispõe também de uma sala de cuidados com leito obstétrico tipo Pré-Parto, Parto e Puerpério (PPP) e berço aquecido para o primeiro atendimento ao recém-nascido; sala de estar para familiares e gestantes com aparelho de TV; sala para oficinas educativas com aparelhos audiovisuais e uma ambulância para os casos que necessitam de remoção para a maternidade de referência.

A equipe é composta por dezoito enfermeiras obstétricas, que prestam assistência em regime de trabalho de 40 horas semanais, oito técnicos de enfermagem, uma nutricionista, uma assistente social e trabalhadores terceirizados para os serviços de cozinha, limpeza, segurança e ambulância.

As variáveis estudadas foram as características sociodemográficas e obstétricas das gestantes: idade materna, cor da pele, estado civil, atividade profissional, escolaridade, paridade e número de consultas de pré-natal.

Em relação ao trabalho de parto, foram investigadas variáveis relativas à presença do acompanhante e às condutas obstétricas adotadas (tipo de dieta, administração de ocitocina e cuidados para o relaxamento e conforto).

As variáveis atinentes ao parto foram posição materna, integridade perineal e índice de Apgar no $1^{\circ}$ e $5^{\circ}$ minutos. No pós-parto, as variáveis estudadas foram as causas das transferências maternas e neonatais para o hospital.

Os critérios de inclusão foram baseados no próprio protocolo assistencial da CPDCF, todos os atendimentos ao parto normal de mulheres com idade igual ou superior a 15 anos, com gestações de feto único, cefálico, com idade gestacional acima da $37^{\mathrm{a}}$ semana e até $42^{\mathrm{a}}$ semana, e classificadas como baixo risco obstétrico na internação para o parto.

Segundo os dados institucionais, foi assistido um total de 500 partos normais na CPDCF no período estudado. Durante a coleta de dados, não houve acesso a 42 prontuários. Portanto, o total de prontuários analisados correspondeu a 458, concernentes aos atendimentos ao parto normal na instituição.

Os dados foram coletados por meio de instrumento estruturado, armazenados em banco de dados informatizado em Excel do Microsoft OfficeÒ2007, tabulados, submetidos ao tratamento estatístico descritivo e apresentados por meio de suas frequências absolutas e relativas. Posteriormente, os resultados foram confrontados com os achados das pesquisas nacionais e internacionais sobre a temática investigada.

\section{RESULTADOS}

As gestantes cujo parto foi atendido na CPDCF eram mulheres jovens, na faixa etária entre 15 e 25 anos de idade (308; 66,6\%). As gestantes com idade superior a 35 anos representaram 2,7\% (12) do total. A maioria (257; 56,3\%) tinha a cor da pele diferenciada da branca, classificadas como parda ou preta.

0 nível de escolaridade dessas mulheres era principalmente o ensino médio completo $(304 ; 42,3 \%)$, seguido do ensino fundamental completo $(122 ; 26,6 \%)$. Apenas uma $(0,2 \%)$ gestante foi registrada como analfabeta. Quanto ao estado civil, predominaram as solteiras (208; 45,5\%) em relação àquelas em união consensual (163;35,6\%) e as casadas (78; 17,1\%). A maioria (309; 67,6\%) não realizava atividade remunerada fora do lar.

Considerando as características obstétricas das parturientes atendidas na CPDCF, a maioria (66\%) delas realizou sete ou mais consultas de pré-natal. Predominaram as gestantes nulíparas, aquelas com nenhuma paridade prévia $(55,0 \%)$, em relação às demais com história de parto anterior (Tabela 1).

Tabela 1. Características obstétricas das parturientes. Casa de Parto, Rio de Janeiro, 20082009. $(n=458)$

\begin{tabular}{lcc}
\hline Caracteristicas & $\mathbf{n}$ & $\%$ \\
\hline Consultas de Pré-natal & 2 & \\
1 a 2 consultas & 26 & 0,4 \\
3 a 4 consultas & 132 & 5,8 \\
5 a 6 consultas & 281 & 28,8 \\
7 ou mais consultas & 17 & 61,3 \\
$\quad$ Não registrado & & 3,7 \\
Paridade & 268 & 58,3 \\
$\quad$ Nenhuma & 134 & 29,3 \\
1 parto anterior & 46 & 10,0 \\
\hline 2 ou mais partos anteriores & 10 & 2,4 \\
\hline Não registrado & & \\
\hline
\end{tabular}


Quanto às condutas obstétricas adotadas, quase a totalidade $(431 ; 94,1 \%)$ das parturientes contou com a presença do acompanhante. Nenhuma gestante teve prescrição de dieta zero, sendo indicada dieta livre em $87,1 \%$ (399) dos atendimentos. A amniotomia foi realizada em $11,1 \%$ (51) das gestantes, e a ocitocina foi administrada em 45,0\% (206) dos atendimentos.
Considerando os cuidados para o conforto e relaxamento da mulher no trabalho de parto, os mais realizados foram os exerć́cios respiratórios $(93,2 \%)$, banho morno $(75,2 \%)$, deambulação $(85,1 \%)$, massagem corporal $(65,7 \%)$ e movimentos pélvicos $(59,9 \%$ ) (Tabela 2).

Tabela 2. Cuidados para o relaxamento e conforto das parturientes. Casa de Parto, Rio de Janeiro. 2008-2009. ( $N=458)$

\begin{tabular}{lcc}
\hline Cuidados & $\mathbf{n}$ & \% \\
\hline Exercícios respiratórios & 416 & 90,8 \\
Deambulação & 364 & 79,5 \\
Banho morno & 306 & 66,8 \\
Massagem corporal & 268 & 58,5 \\
Música ambiente & 265 & 57,9 \\
Movimentos pélvicos & 257 & 56,1 \\
Proteção perineal & 237 & 51,7 \\
Óleo essencial & 112 & 24,5 \\
Essência aromática & 98 & 21,4 \\
Bola de Bobath & 32 & 7,0 \\
Técnica do rebozo & 22 & 4,8 \\
Banquinho de parto & 18 & 3,9 \\
\hline
\end{tabular}

A posição materna no parto foi variada, com maior frequência da posição lateral $(180 ; 39,3 \%)$, vertical $(158 ; 34,5 \%)$ e em cócoras $(48 ; 10,5 \%)$. A posição horizontal foi adotada por apenas 4,1\% (19) parturientes.

Para a maioria das parturientes $(63,0 \%)$ houve a ocorrência de laceração perineal de primeiro grau, seguida de casos em que o períneo se manteve íntegro $(21,2 \%)$ e de laceração de segundo grau (12,4\%). Não houve ocorrência de trauma perineal grave, lacerações de períneo de terceiro e quarto graus. A episiotomia foi realizada em apenas 2,4\% das parturientes (Tabela 3 ).

Tabela 3. Condição do períneo no parto normal. Casa de Parto, Rio de Janeiro, 2008 -

2009

\begin{tabular}{lcc}
\hline Condição do períneo & n & \% \\
\hline Laceração de $1^{\circ}$ grau & 289 & 63,0 \\
Íntegro & 97 & 21,2 \\
Laceração de $2^{\circ} \mathrm{grau}$ & 57 & 12,4 \\
Episiotomia & 11 & 2,4 \\
Sem registro & 5 & 1,0 \\
Total $^{*}$ & 459 & 100 \\
\hline
\end{tabular}

(*) Em um caso houve a ocorrência simultânea de episiotomia e laceração de períneo.

Em relação aos recém-nascidos, a grande maioria nasceu em boas condições e obteve índice de Apgar entre 9 e 10 tanto no primeiro minuto $(76,9 \%)$ quanto no quinto minuto $(95,4 \%)$.
Houve apenas um caso $(0,2 \%)$ de asfixia neonatal, índice de Apgar abaixo de 7 no quinto minuto (Tabela 4).

Tabela 4. Índice de Apgar dos recém-nascidos. Casa de Parto, Rio de Janeiro, 2008-2009

\begin{tabular}{lcccc}
\hline Índice de Apgar & \multicolumn{2}{c}{ Primeiro minuto } & \multicolumn{2}{c}{ Quinto minuto } \\
\hline & $\mathbf{n}$ & $\%$ & $\mathbf{n}$ & $\%$ \\
Abaixo de 7 & 22 & 4,8 & 1 & 0,2 \\
$7-8$ & 84 & 18,3 & 20 & 4,4 \\
$9-10$ & 352 & 76,9 & 437 & 95,4 \\
Total & 458 & 100 & 458 & 100 \\
\hline
\end{tabular}


Foram transferidos para o hospital 13 puérperas (2,8\%) e 39 neonatos (8,5\%). A principal causa de remoção materna foi a permanência de restos placentários ou de membranas ovulares (8), seguida da hipotonia uterina (2), hipertensão arterial (2) e infecção urinária (1). As transferências dos neonatos para o hospital tiveram como principal causa 0 desconforto respiratório (14). As demais transferências foram motivadas pelo baixo peso ao nascer (7), hipoglicemia (4), infecção (4) e icterícia (4). Não houve a ocorrência de óbito materno e neonatal no período do estudo (Tabela 5).

Tabela 5. Causas das transferências maternas no pós-parto e dos neonatos para o hospital. Casa de Parto, Rio de Janeiro, 2008-2009

\begin{tabular}{|ccc|}
\hline Causas & $n$ & $\%$ \\
\hline Transferências maternas & & \\
\hline Restos placentários & 8 & 61,5 \\
\hline Hipotonia uterina & 2 & 15,4 \\
\hline Hipertensão arterial & 2 & 15,4 \\
\hline Infecção urinária & 1 & 7,7 \\
\hline Total & 13 & 100 \\
Transferências dos neonatos & & \\
Desconforto respiratório & 14 & 35,9 \\
\hline Baixo peso ao nascer & 7 & 17,8 \\
\hline Hipoglicemia & 4 & 10,3 \\
\hline Infecção & 4 & 10,3 \\
\hline Icterícia & 4 & 10,3 \\
\hline Má formação congênita & 3 & 7,7 \\
\hline Outras causas & 3 & 7,7 \\
\hline Total & 39 & 100 \\
\hline
\end{tabular}

\section{DISCUSSÃO}

O perfil sociodemográfico das parturientes na CPDCF é semelhante àqueles de mulheres que são assistidas em CPN. A predominância de mulheres com menos de 25 anos e que não exercem atividade laboral remunerada foi também observado em CPN brasileiros ${ }^{6-7}$. Em CPN peri-hospitalar, os atendimentos foram principalmente de mulheres que cursaram oito ou mais anos de estudo e eram casadas ou unidas ${ }^{7}$.

A nuliparidade e a realização de sete ou mais consultas de pré-natal também foram características obstétricas mais frequentes entre as mulheres assistidas em $\mathrm{CPN}^{7-8}$. Nos centros de parto australianos, o perfil obstétrico é diferenciado, $65,1 \%$ mulheres tinham história prévia de uma ou mais paridade 9 .

A presença do acompanhante é um direito legal previsto na Lei Federal 11.108/2005, que contribui na vivência processo do parto pela mulher. No entanto, ainda há barreiras institucionais nas maternidades brasileiras na garantia do exercício desse direito pelas gestantes. Em 2006, a Pesquisa Nacional de Demografia e Saúde da Criança e da Mulher identificou que menos de $10 \%$ das mulheres usufruíram esse direito nas instituições do SUS. No sistema privado este percentual foi de $35 \%{ }^{10}$.

Estudo em CPN peri-hospitalar também foi observada elevada proporção $(92,2 \%)$ de mulheres que contaram com acompanhantes no trabalho de parto, semelhante ao encontrado na CPDCF.? Tal achado denota que as gestantes usuárias dos centros de parto têm seus direitos respeitados, como preconizado na legislação e política de saúde brasileira.
0 acesso a líquidos e alimentação é necessário para repor as fontes de energia, garantir o bem-estar fetal e materno e prevenir a desidratação e a cetose. Revisão sistemática evidenciou que não existe justificativa para restringir líquidos e alimentos para as parturientes classificadas como baixo risco ${ }^{11}$. Oferecimento de dieta oral às parturientes também foi verificada em CPN peri-hospitalar?. Portanto, a hidratação e a nutrição da mulher durante o trabalho de parto fisiológico são condutas assistenciais fundamentadas em evidências cientificas.

A utilização de ocitocina durante o trabalho de parto está relacionada com o aumento da sensação dolorosa, podendo causar uma postura mais restrita ao leito, maior predisposição à ocorrência de hiperestimulação uterina e alteração na frequência cardíaca fetal ${ }^{12}$. A proporção da administração desse fármaco na CPDCF foi semelhante ao observado em CPN intrahospitalar, correspondendo a 44,5\% dos atendimentos, e maior que a taxa de 23,5\% em outro CPN peri-hospitalar ${ }^{8-7}$. Estas elevadas taxas de utilização de ocitocina devem ser objeto de investigações para elucidar suas causas e fatores predisponentes.

Em relação à prática de amniotomia, a frequência dessa intervenção na CPDCF $(11,1 \%)$ foi consideravelmente menor que a verificada em pesquisas em CPN brasileiros, que encontraram os percentuais de $62,3 \%$ e $75,1 \% 7^{7-8}$. Tal diferença pode estar relacionada ao protocolo assistencial da instituição, que restringe essa prática e recomenda a realização de amnioscopia na admissão ou no curso do trabalho de parto das gestantes com o colo dilatado e membranas amnióticas 
íntegras. Este exame visa à detecção precoce de mecônio no líquido amniótico. Nos casos de líquido amniótico tinto de mecônio, as parturientes são removidas para o hospital.

Os cuidados mais realizados na CPDCF para o relaxamento e conforto das gestantes no trabalho de parto foram os exercícios respiratórios, deambulação, banho morno e massagem corporal. Em CPN peri-hospitalar, estes cuidados também foram variados, tais como: banho de aspersão (71\%), deambulação (47,6\%), massagem (29,8\%), banho de imersão (21,9\%), entre outros ${ }^{7}$.

Em relação à posição materna no parto, as mais frequentes na CPDCF foram as posições lateral, vertical e em cócoras. Estudo realizado em CPN intra-hospitalar identificou a predominância (52\%) da posição vertical, seguida da litotômica $(31 \%)$ e a vertical $(3,7 \%)^{8}$.

Uma revisão sistemática evidenciou que a posição lateral ou vertical, em comparação com as posições supina e a litotômica, estão associadas a redução do segundo estágio do trabalho de parto, de episiotomias, dos relatos de dor intensa, menor ocorrência de frequência cardíaca fetal anormal e aumento das lacerações perineais de segundo grau. Os autores recomendam que as mulheres devam adotar a posição mais confortável no parto ${ }^{13-14}$.

$\mathrm{Na}$ CPDCF, em $21,2 \%$ das parturientes, o períneo manteve-se íntegro, em 12,4\% teve laceração de segundo grau, e a taxa de episiotomia foi de $2,4 \%$. Pesquisas em CPN brasileiros verificaram a ocorrência diferenciada de lacerações de segundo grau, 7,5\% e 17,5\% ${ }^{12-15}$. Em Centros de Parto da Austrália, 25,6\% de parturientes permaneceram com períneo íntegro. Nas demais mulheres atendidas houve a realização de episiotomia em $5,4 \%$ dos partos e a ocorrência de laceração de períneo de $1^{\circ}$ e $2^{\circ}$ graus foi de $12,6 \%$ e $39,4 \%$, respectivamente ${ }^{16}$.

No Brasil, o Ministério da Saúde não preconiza um limite aceitável de episiotomia no parto, apesar de recomendar a utilização restrita desse procedimento. Por outro lado, estudos são controversos em relação às taxas aceitáveis de episiotomia, que variam de 10,20 a $30 \%{ }^{17}$.

A proporção de episiotomia na CPDCF é semelhante aos praticados nos CPN internacionais. Estudos encontraram menores taxas de episiotomia no segmento de mulheres cujo parto ocorreu em centros de parto ou no domicílio, correspondendo a 3,8\% no Canadá; 7,8\% na Suécia; 15,7\% na Alemanha e 17,6\% nos Estados Unidos. ${ }^{12}$ Em Centros de Parto australianos, a episiotomia foi realizada em $7,7 \%$ das mulheres. ${ }^{8}$ No Reino Unido, foi verificado o percentual de $5 \%$ nesses centros, enquanto nos hospitais foi de $18,9 \%$ de episiotomia $^{18}$.

Os resultados para asfixia neonatal da CPDCF apresentam tendência semelhante aos Centros de Parto brasileiros e internacionais. Em relação aos resultados neonatais, estudo em CPN intra-hospitalar brasileiro encontrou o percentual de $1 \%$ dos recém-nascidos que receberam um valor de Apgar abaixo de 7 no quinto minuto de vida ${ }^{18}$. Esses casos ocorreram em 0,6\% dos nascidos vivos nos Centros de Parto da Austrália ${ }^{16}$.

As transferências maternas no pós-parto na CPDCF representaram 2,8\% dos atendimentos. Uma pesquisa que analisou 2.117 atendimentos em CPN intra-hospitalar, entre janeiro 2002 e julho 2003, encontrou a taxa de transferência materna de $11,4 \%$. As transferências predominaram no período intraparto, e no pós-parto corresponderam a $0,9 \%$. Após o parto, as principais causas das remoções maternas foram a ocorrência de hemorragia $(4,6 \%)$, pressão arterial $\geq 140 / 90 \mathrm{mmHg}(1,7 \%)$ e febre $(1,2 \%)^{6}$.

$\mathrm{Na}$ CPDCF as transferências neonatais (8,5\%) também foram mais frequentes que as observadas em estudos realizados em CPN brasileiros. Em CPN intra-hospitalar identificou-se que 3,8\% dos recém-nascidos necessitaram de transferência para a Unidade Neonatal ${ }^{6}$. Em CPN peri-hospitalar, os neonatos que necessitaram de internação em unidade neonatal corresponderam a 1,4\% dos 991 atendimentos analisados? Em Centros de Partos australianos, esse percentual foi de 6,9\% em 22.232 nascimentos $^{16}$.

$\mathrm{Na}$ CPDCF, as causas das remoções neonatais para o hospital foram semelhantes ao encontrado em pesquisa em CPN intra-hospitalar, que teve como principais motivos das remoções a depressão ou desconforto respiratório (36,3\%), infecção (22,5\%), icterícia (18,8\%), anomalias congênitas $(6,3 \%)$, prematuridade $(5,0 \%)$ e tamanho pequeno ou grande para idade gestacional $(3,7 \%)^{6}$.

Deve ser ressaltado que a assistência materna e neonatal na CPDCF encontra-se sob responsabilidade integral das enfermeiras obstétricas. Em caso de necessidade de avaliação médica, as mulheres e os neonatos são prontamente removidos para o hospital. Essa conduta é prevista no protocolo assistencial da CPDCF e visa garantir a continuidade da linha de cuidado nas situações em que é necessária assistência em maior nível complexidade de atenção, assegurando o bemestar materno e dos recém-nascidos.

\section{CONCLUSÃO}

As parturientes atendidas na CPDCF são mulheres jovens, que contaram com a presença do acompanhante e tiveram uma assistência humanizada e segura no momento do parto e do nascimento de seus filhos.

A maioria dos resultados maternos e neonatais revelou que são estabelecidas práticas assistenciais pautadas nas evidências científicas e que consideram a cidadania das mulheres, promovem o bem-estar materno e neonatal, e são semelhantes aos achados das pesquisas nacionais e internacionais em Centros de Parto Normal.

Esses resultados sugerem que as Casas de Parto podem dar significativas contribuições às ações de qualificação e humanização da assistência obstétrica no Sistema de Saúde brasileiro. 
Cabe mencionar que há limitações na presente pesquisa relacionadas ao pequeno quantitativo dos atendimentos analisados na CPDCF e ao desenho metodológico utilizado, que não permite inferências.

Novas investigações são necessárias para elucidar as questões não esclarecidas pelo estudo e avançar na produção de dados pertinentes à assistência de enfermagem obstétrica nesse cenário de atenção.

\section{REFERÊNCIAS}

1.Phillippi JC, Alliman J, Bauer K. The American Association of Birth Centers: History, membership, and current initiatives. J Midwifery Womens Health. 2009 Sep/0ct; 54(5): 387-92.

2.Devane D, Murphy-Lawless J, Begley CM. Childbirth policies and practices in Ireland and the journey towards midwifery-led care. Midwifery. 2007 Mar; 23(1): 92-101.

3.Borquez HA, Wiegers TA. A comparison of labour and birth experiences of women delivering in a birthing centre and at home in the Netherlands. Midwifery. 2006 Dec; 22(4): 339-47.

4. Coyle KL, Hauck Y, Percival P, Kristjanson L. Normality and collaboration: mothers' perceptions of birth centre versus hospital care. Midwifery. 2001 Sep; 17(3): 182-93.

5.Portaria n. 985 de 05 de agosto de 1999. Cria o Centro de Parto Normal-CPN, no âmbito do Sistema Único de saúde/SUS, para o atendimento à mulher no período gravídico-pueperal. Diário Oficial da República Federativa do Brasil, Brasília(DF), de 6 agosto de 1999: seção 1: p. 512

6.Campos SEV, Lana FCF. Resultados da assistência ao parto no Centro de Parto Normal Dr. David Capistrano da Costa Filho em Belo Horizonte, Minas Gerais, Brasil. Cad. saúde pública. 2007 jun; 23(6): 1349-59.

7. LoboSF, OliveiraSMJV, SchneckA, Silva FMB, Bonadio IC, Riesco MLG. Resultados maternos e neonatais em Centro de Parto Normal perihospitalar na cidade de São Paulo, Brasil. Rev. Esc. Enferm. USP. 2010 set; 44(3): 812-8.

8.Schneck CA, Riesco MLG. Intervenções no parto de mulheres atendidas em um centro de parto normal intra-hospitalar. REME rev. min. enferm. 2006 jul/set; 10(3): 240-6.

9.Tracy SK, et al. Birth centers in Australia: a national population-based study of perinatal mortality associated with giving birth in a birth center. Birth. 2007 Sep; 34(3): 194-201.

10.Ministério da Saúde (BR). Pesquisa Nacional de Demografia e Saúde da Criança e da Mulher - PNSD 2006: Relatório. Brasília(DF): Ministério da Saúde; 2008.

11.Singata M, Tranmer J, Gyte GML. Restricting oral fluid and food intake during labour. Cochrane Database Syst Rev. 2010 Jan 20;(1): CD 003930.
12.Svärdby K, Nordström L, Sellström E. Primiparas with or without oxytocin augmentation: a prospective descriptive study. J Clin Nurs. 2007; 16(1): 179-84.

13.Mamede FV, Almeida AM, Souza L, Nakano AMS, Gomes FA, Panobianco MS. 0 efeito da deambulação na duração da faze ativa do trabalho de parto. Esc Anna Nery. 2007 set; 11(3): 466-71

14.Lawrence A, Lewis L, Hofmeyr GJ, Dowswell T, Styles C. Maternal positions and mobility during first stage labour. Cochrane Database Syst Rev. 2009;15(2): CD003934

15. Costa SCA, Riesco MLG. A comparison of "hands off" versus "hands on" techniques for decreasing perineal lacerations during birth. J Midwifery Womens Health. 2006 Mar/Apr; 51(2): 106-11.

16.Laws PJ, Tracy SK, Sullivan EA. Perinatal outcomes of women intending to give birth in Birth Centers in Australia. Birth. 2010 Mar; 37(1): 28-36.

17.Graham ID, Carroli G, Davies C, Medves JM. Episiotomy rates around the world: An update. Birth. 2005 Sep; 32(3): 219-23.

18.Walsh D, Downe SM. Outcomes of free-standing, midwife-led birth centers: A structured review. Birth. 2004 Sep; 31(3): 222-9. 